\title{
Extended Frequency-Directed Run-Length Code with Improved Application to System-on-a-Chip Test Data Compression
}

\author{
Aiman H. El-Maleh and Raslan H. Al-Abaji \\ King Fahd University of Petroleum and Minerals, Dhahran 31261, Saudi Arabia \\ Email: \{aimane, raslan\}@ccse.kfupm.edu.sa
}

\begin{abstract}
One of the major challenges in testing a System-on-aChip (SOC) is dealing with the large test data size. To reduce the volume of test data, several test data compression techniques have been proposed. Frequencydirected run-length (FDR) code is a variable-to-variable run length code based on encoding runs of 0's. In this work, we demonstrate that higher test data compression can be achieved based on encoding both runs of O's and 1 's. We propose an extension to the FDR code and demonstrate by experimental results its effectiveness in achieving higher compression ratio.
\end{abstract}

\section{INTRODUCTION}

Advances in VLSI technology have resulted in a change in the design paradigm where complete systems containing millions of transistors are integrated on a single chip. As the complexity of systems-on-a-chip continues to increase, the difficulty and cost of testing such chips is increasing rapidly [1-2]. One of the challenges in testing system-on-a-chip is dealing with the large size of test data that must be stored in the tester memory and transferred between the tester and the chip under test. The cost of automatic test equipment (ATE) increases significantly with the increase in their speed, channel capacity, and memory. Thus, to reduce the testing time and cost, it is necessary to reduce the volume of test data.

Test data reduction can be achieved by both test compaction [3-7] and compression [8-14]. Several test data compression techniques have been proposed in the literature. In [8], statistical coding is used for encoding test data based on a modified version of Huffman coding. In [9], efficient test data compression is achieved based on partitioning the test data into two-dimensional blocks and encoding each block separately based on geometric shapes. Another technique proposed in [10] uses what is called variable-to-block run-length coding. In this technique, a code word is used to encode a block of data based on the number of zeros followed by a one in that block. This technique is used for compressing fully specified test data that feeds a cyclical scan chain. A cyclical scan chain is used to decompress this data and transfer it to the "test scan chain". Golomb code is a variable-to-variable run-length code that is used in [11] to enhance the scheme described above. It divides the runs into groups each is of size $m$. The number of groups is determined by the length of the longest run, and the group size $m$ is dependent on the distribution of test data. Another enhancement to the work done in [10] and [11] was proposed in [12]. It uses frequency-directed runlength (FDR) code, which is another variable-to-variable coding technique. It is designed based on the observation that the frequency of runs decreases with the increase in their lengths. Hence, assigning smaller code words to runs with small length and larger code words to those with larger length could result in higher test data compression.

The techniques in [10-12] are all based on encoding only runs of 0 's. This was motivated based on the idea that encoding the difference vectors instead of the actual test vectors may reduce the number of 1's in the encoded data. However, it was demonstrated in [12] that, in general, better test data compression results are achieved, based on both FDR and Golomb codes, by encoding the actual test vectors. Based on test data analysis, we have observed that the frequency of runs of 1's is as significant as runs of 0 's, for many of the circuits. This suggests that encoding both runs of 0 's and 1's could result in higher test data compression. In this work, we propose an extension to the FDR code to encode the test data based on encoding both types of runs.

\section{FREQUENCY-DIRECTED RUN-LENGTH (FDR) CODE}

Many of the test data compression techniques are based on run-length coding. A run is a consecutive sequence of equal symbols. A sequence of symbols can be encoded using two elements for each run; the repeating symbol and the number of times it appears in the run. Frequency-directed run-length (FDR) code is a variable-to-variable coding technique based on encoding runs of 0 's. In FDR code, the prefix and the tail of any codeword are of equal size. In any group $A_{i}$, the prefix is of size $i$ bits. The prefix of a group is the binary representation of the run length of the first member of 
Table 1. FDR code.

\begin{tabular}{|c|c|c|c|c|}
\hline Group & $\begin{array}{c}\text { Run } \\
\text { Length }\end{array}$ & \begin{tabular}{|l|} 
Group \\
Prefix
\end{tabular} & Tail & Code Word \\
\hline \multirow[t]{2}{*}{ A1 } & 0 & \multirow{2}{*}{0} & 0 & 00 \\
\hline & 1 & & 1 & 01 \\
\hline \multirow{4}{*}{ A2 } & 2 & \multirow{4}{*}{10} & 00 & 1000 \\
\hline & 3 & & 01 & 1001 \\
\hline & 4 & & 10 & 1010 \\
\hline & 5 & & 11 & 1011 \\
\hline \multirow{8}{*}{ A3 } & 6 & \multirow{8}{*}{110} & 000 & 110000 \\
\hline & 7 & & 001 & 110001 \\
\hline & 8 & & 010 & 110010 \\
\hline & 9 & & 011 & 110011 \\
\hline & 10 & & 100 & 110100 \\
\hline & 11 & & 101 & 110101 \\
\hline & 12 & & 110 & 110110 \\
\hline & 13 & & 111 & 110111 \\
\hline
\end{tabular}

that group. When moving from group $A_{i}$ to group $A_{i+1}$, the length of the code words increases by two bits, one for the prefix and one for the tail. Runs of length $i$ are mapped to group $A_{j}$, where $j=\left\lceil\log _{2}(i+3)\right\rceil-1$. The size of the $i$ 'th group is equal to $2^{i}$, i.e., group $A_{i}$ contains $2^{i}$ members. The FDR code for the first three groups is shown in Table 1.

\section{TEST DATA ANALYSIS}

Based on test data analysis, it has been observed that test sets contain a large number of runs of 1's in addition to runs of 0's. By considering both types of runs, the total number of runs will decrease, which could result in higher test data compression.

To support this observation, we have analyzed test data for the largest ISCAS 85 and full-scanned versions of ISCAS 89 circuits. We have used the test sets generated by MinTest [5], using both static and dynamic compaction. Test sets generated by dynamic compaction option have the letter $d$ appended in their name. All the test sets used achieve $100 \%$ fault coverage of the detectable faults in each circuit. Test sets generated based on static compaction were relaxed, as this has the advantage of keeping unnecessary assignments as X's, which enables higher compression.

Given a relaxed test set, techniques based on encoding only runs of 0's fill all the X's by 0's to reduce the number of runs that need to be encoded. However, to encode both runs of 0 's and 1's in a test set, X's are filled by 1's if they are bounded by 1's from both sides, otherwise they are filled by 0's. This results in a reduction in the total number of runs that need to be encoded.

Table 2 shows the analysis of the number of runs on the used test sets. The first column indicates the circuit name. The second column shows the number of runs of 0 's in the test set assuming that only runs of 0 's will be encoded. The third, fourth, and fifth columns indicate the number of runs of 0 's, runs of 1 's, and the total number of runs, respectively, assuming that both types of runs
Table 2. Analysis of number of runs in test data.

\begin{tabular}{|c|c|c|c|c|c|}
\cline { 3 - 6 } \multicolumn{2}{c|}{} & $\begin{array}{c}\text { Encoding } \\
\text { 0 Runs }\end{array}$ & \multicolumn{3}{|c|}{$\begin{array}{c}\text { Encoding } \\
\text { 0 }\end{array}$} \\
\hline Circuit 1 Runs \\
\hline c2670 & $\begin{array}{c}\text { Original } \\
\text { Bits }\end{array}$ & 0 Runs & 0 Runs & 1 Runs & $\begin{array}{c}\text { Total } \\
\text { Runs }\end{array}$ \\
\hline c5315 & 6586 & 1677 & 505 & 414 & 919 \\
\hline c7552 & 15111 & 2695 & 652 & 1111 & 1763 \\
\hline s13207 & 163100 & 4804 & 2615 & 1157 & 3772 \\
\hline s15850 & 57434 & 4635 & 2514 & 1106 & 3620 \\
\hline s35932 & 21156 & 7554 & 1236 & 1071 & 2307 \\
\hline s38417 & 113152 & 20970 & 5331 & 3761 & 9092 \\
\hline s5378 & 20758 & 2915 & 1072 & 806 & 1878 \\
\hline s9234 & 25939 & 3843 & 1770 & 980 & 2750 \\
\hline s13207d & 165200 & 5021 & 2581 & 1210 & 3791 \\
\hline s15850d & 76986 & 5329 & 2644 & 1202 & 3846 \\
\hline s35932d & 28208 & 10018 & 235 & 346 & 581 \\
\hline s38417d & 164736 & 29473 & 5773 & 4834 & 10607 \\
\hline s38584d & 199104 & 16814 & 7585 & 4074 & 11659 \\
\hline s5378d & 23754 & 3537 & 1237 & 1001 & 2238 \\
\hline s9234d & 39273 & 4816 & 2347 & 1212 & 3559 \\
\hline
\end{tabular}

will be encoded. As can be seen from the table, for most of the circuits, the number of runs of 1's is as significant as the number of runs of 0 's. For all the circuits, the total number of runs decreases and for some circuits the reduction is significant.

Figures 1, 2, and 3 show the frequency of both runs of 0 's and runs of 1's for test sets of the circuits: s15850, s9234, and s35932d, respectively. As can be seen from the figures, the frequency of runs of 1's follow a similar shape to that of runs of 0 's, although with a smaller

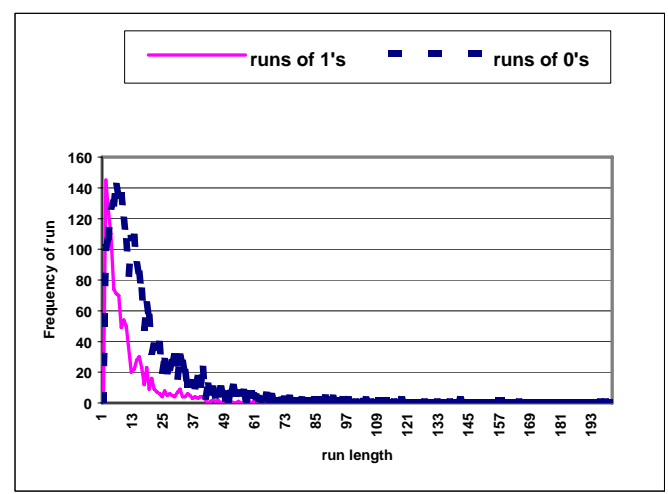

Figure 1. Distribution of runs of 0's and 1's for circuit s15850.

magnitude. For the circuit in Figure 1, it can be observed that there are more runs of 1's than 0 's for run length $<5$, but for run length $>5$ there are more runs of 0 's. For the circuit in Figure 2, we can see that runs of 0's with any length are on the average more that the runs of 1's with the same length. For the circuit in Figure 3, it can be observed that runs of 1's of small and large run length are more than those of 0's. But for middle run length ranges, the number of both 0 and 1 runs is comparable. 


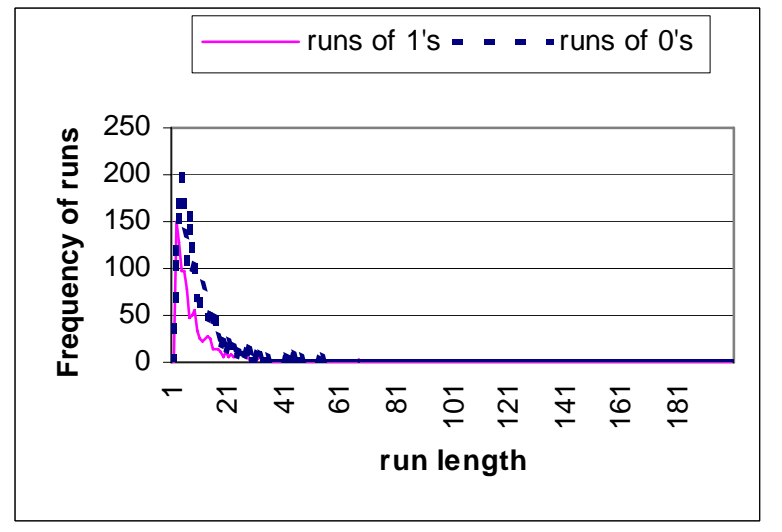

Figure 2. Distribution of runs of 0's and 1's for circuit s9234.

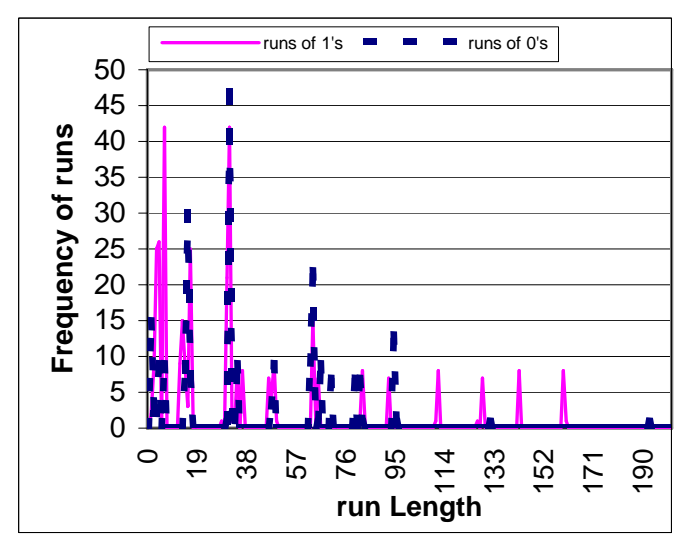

Figure 3. Distribution of runs of 0's and 1's for circuit s35932d.

\section{EXTENDED FDR (EFDR) CODE}

To encode both runs of 0's and 1's, we extend the FDR code based on adding an extra bit to the beginning of a code word to indicate the type of run. If the bit is 0 , this indicates that the code word is encoding a run of type 0 , otherwise it encodes a run of type 1 . This code, called Extended FDR (EFDR), is shown in Table 3. It should be observed that this code is a direct extention to the FDR code shown in Table 1. However, in this code we do not have run length of size 0 . This is because we are encoding both runs of 0 's and runs of 1 's. Note that runs of 0 's are strings of 0 's followed by a 1 , while runs of 1 's are strings of 1's followed by a 0 , i.e. runs of 1 's of length $i$ are the complement of runs of 0's of the same length, and vice versa. As with FDR code, in this code when moving from group $A_{i}$ to group $A_{i+1}$, the length of code words increases by two bits, one for the prefix and one for the tail. Runs of length $i$ are mapped to group $A_{j}$, where $j=\left\lceil\log _{2}(i+2)\right\rceil-1$. The size of the $i$ 'th group is equal to $2^{i+1}$, i.e., group $A_{i}$ contains $2^{i+1}$ members.

To illustrate the use of this code, let us consider an example. Consider the test $\mathrm{T}=\{0110001111111000000001\}$, of size 22 bits. The
Table 3. Extended FDR (EFDR) code.

\begin{tabular}{|c|c|c|c|c|c|}
\hline Group & $\begin{array}{c}\text { Run } \\
\text { Length }\end{array}$ & $\begin{array}{l}\text { Group } \\
\text { Prefix }\end{array}$ & Tail & $\begin{array}{l}\text { Code Word } \\
\text { Runs of 0's }\end{array}$ & $\begin{array}{l}\text { Code Word } \\
\text { Runs of 1's }\end{array}$ \\
\hline \multirow[t]{2}{*}{ A1 } & 1 & \multirow{2}{*}{0} & 0 & 000 & 100 \\
\hline & 2 & & 1 & 001 & 101 \\
\hline \multirow{4}{*}{ A2 } & 3 & \multirow{4}{*}{10} & 00 & 01000 & 11000 \\
\hline & 4 & & 01 & 01001 & 11001 \\
\hline & 5 & & 10 & 01010 & 11010 \\
\hline & 6 & & 11 & 01011 & 11011 \\
\hline \multirow{8}{*}{ A3 } & 7 & \multirow{8}{*}{110} & 000 & 0110000 & 1110000 \\
\hline & 8 & & 001 & 0110001 & 1110001 \\
\hline & 9 & & 010 & 0110010 & 1110010 \\
\hline & 10 & & 011 & 0110011 & 1110011 \\
\hline & 11 & & 100 & 0110100 & 1110100 \\
\hline & 12 & & 101 & 0110101 & 1110101 \\
\hline & 13 & & 110 & 0110110 & 1110110 \\
\hline & 14 & & 111 & 0110111 & 1110111 \\
\hline
\end{tabular}

number of 0 runs in this test is 10 . However, the number of both 0 and 1 runs is 5. Encoding this test using FDR code results in the encoded test $\mathrm{T}_{\mathrm{FDR}}=\{010010010000$ $00000000110010\}$ of size 26 bits. Thus, for this example the number of bits needed to encode the test data using FDR code is more than the actual size of the original test data. However, encoding this test using EFDR code, we obtain the encoded test $T_{\text {EFDR }}=\{000100$ $001110110110000\}$, of size 21 bits. Obviously, for this example EFDR code outperforms FDR code. Note that FDR code suffers whenever we have runs of 1 's, as each 1 bit will be encoded by a separate 0 run of length 0 .

\section{EXPERIMENTAL RESULTS}

Table 4 compares the compression results using the FDR and EFDR code. The first column shows the circuit name and the second column shows the size of the test set in bits. The third and fourth columns show the number of compressed bits using FDR and EFDR codes, respectively. The last two columns indicate the respective compression ratios. The compression ratio is computed as:

$$
\text { Comp. Ratio }=\frac{\# \text { Original Bits }-\# \text { Compressed Bits }}{\# \text { Original Bits }} X 100
$$

As can be seen from the table, significant improvements in the compression ratio are obtained for some of the circuits. Consider for example the circuit s35932. For the first test set of this circuit, the compression ratio improves from 3.99\% using FDR to $45.63 \%$ using EFDR code. For the second test set of the same circuit, the compression ratio increases from $19.36 \%$ using FDR to 80.31\% using EFDR code. This result is not surprising as based on the statistics for this circuit given in Table 2, the total number of runs reduces significantly when both types of runs are used versus using only 0 runs. Similarly, significant increase in the compression ratio is obtained for the test sets c2670, c5315, s38417, and s38417d. For 
Table 4. Compression results of FDR \& EFDR codes.

\begin{tabular}{|c|c|c|c|c|c|}
\hline Circuit & $\begin{array}{c}\text { Original } \\
\text { Bits }\end{array}$ & $\begin{array}{c}\text { FDR } \\
\text { Bits }\end{array}$ & $\begin{array}{c}\text { EFDR } \\
\text { Bits }\end{array}$ & $\begin{array}{c}\text { FDR } \\
\text { CR }\end{array}$ & $\begin{array}{c}\text { EFDR } \\
\text { CR }\end{array}$ \\
\hline c2670 & 10252 & 5760 & 4807 & 43.82 & $\mathbf{5 3 . 1 1}$ \\
\hline c5315 & 6586 & 5238 & 4700 & 20.47 & $\mathbf{2 8 . 6 4}$ \\
\hline c7552 & 15111 & 9500 & 8843 & 37.13 & $\mathbf{4 1 . 4 8}$ \\
\hline s13207 & 163100 & 34608 & 33637 & 78.78 & $\mathbf{7 9 . 3 8}$ \\
\hline s15850 & 57434 & 24992 & 25105 & $\mathbf{5 6 . 4 9}$ & 56.29 \\
\hline s35932 & 21156 & 20312 & 11502 & 3.99 & $\mathbf{4 5 . 6 3}$ \\
\hline s38417 & 113152 & 70536 & 53914 & 37.66 & $\mathbf{5 2 . 3 5}$ \\
\hline s5378 & 20758 & 11032 & 10210 & 46.85 & $\mathbf{5 0 . 8 1}$ \\
\hline s9234 & 25939 & 16912 & 16127 & 34.80 & $\mathbf{3 7 . 8 3}$ \\
\hline s13207d & 165200 & 30880 & 29992 & 81.31 & $\mathbf{8 1 . 8 5}$ \\
\hline s15850d & 76986 & 26016 & 24643 & 66.21 & $\mathbf{6 7 . 9 9}$ \\
\hline s35932d & 28208 & 22746 & 5554 & 19.36 & $\mathbf{8 0 . 3 1}$ \\
\hline s38417d & 164736 & 93452 & 64962 & 43.27 & $\mathbf{6 0 . 5 7}$ \\
\hline s38584d & 199104 & 77798 & 73853 & 60.93 & $\mathbf{6 2 . 9 1}$ \\
\hline s5378d & 23754 & 12356 & 11419 & 47.98 & $\mathbf{5 1 . 9 3}$ \\
\hline s9234d & 39273 & 22148 & 21250 & 43.61 & $\mathbf{4 5 . 8 9}$ \\
\hline
\end{tabular}

all the test sets except one, using EFDR code achieves higher compression ratio.

For test data decompression based on EFDR code, the decoder design follows a direct extention of the FDR decoder proposed in [12].

\section{CONCLUSION}

In this work, we have proposed an extension to the recently proposed FDR code, namely Extended FDR (EFDR) code. The proposed technique is based on encoding both runs of 0 's and 1's as opposed to encoding only runs of 0's. Based on experimental results on ISCAS benchmark circuits, it has been demonstrated that the proposed EFDR code outperformed FDR code and resulted in significant increase in test data compression ratio for several circuits, improving the compression ratio from $19.36 \%$ to $80.31 \%$ for one of the benchmark circuits.

\section{ACKNOWLEDGMENT}

This project is supported by King Fahd University of Petroleum \& Minerals under project FT2000/07.

\section{REFERENCES}

[1] R. Chandramouli, and S. Pateras, "Testing Systems on a Chip,” IEEE Spectrum, pp. 42-47, Nov. 1996.

[2] Y. Zorian, E.J. Marinissen, and S. Dey, "Testing EmbeddedCore Based System Chips,” Proc. of Int. Test Conf., pp. 130143, 1998.
[3] M. Schulz, E. Trischhler, and T. Sarfert, "SOCRATES: A Highly Efficient Automatic Test Pattern Generation System," IEEE Trans. Computer-Aided Design, pp. 126-137, Jan. 1988.

[4] I. Pomeranz, L. Reddy, and S. Reddy, "COMPACTEST: A Method to Generate Compact Test Sets for Combinational Circuits,” Proc. of Int. Test Conference, pp. 194-203, 1991.

[5] I. Hamzaoglu and J. H. Patel, "Test Set Compaction Algorithms for Combinational Circuits", Proc. Int. Conf. Computer-Aided Design, pp. 283-289, Nov. 1998.

[6] J. Chang and C. Lin, "Test Set Compaction for Combinational Circuits," IEEE Trans. Computer Aided Design, pp. 1370-1378, Nov. 1995.

[7] S. Kajihara, I. Pomeranz, K. Kinoshita, and S. Reddy, "CostEffective Generation of Minimal Test sets for Stuck-at Faults in Combinational Circuits,” IEEE Trans. Computer Aided Design, pp. 1496-1504, Dec. 1995.

[8] A. Jas, J.G. Dastidar and N.A. Touba, "Scan Vector Compression/ Decompression Using Statistical Coding," Proc. of IEEE VLSI Test Symp., pp. 114-120, 1999.

[9] A. El-Maleh, S. Al-Zahir, and E. Khan, "A GeometricPrimitives-Based Compression Scheme for Testing Systems-ona-Chip,” Proc. of IEEE VLSI Test Symp., pp. 54-59, 2001.

[10] A. Jas and N.A. Touba, "Test Vector Decompression via Cyclical Scan Chains and its Application to Testing Core-Based Designs,” Proc. of Int. Test Conf., pp. 458-464, 1998.

[11] A. Chandra and K. Chakrabarty, "Test Data Compression for System-On-a-Chip using Golomb Codes,” Proc. of IEEE VLSI Test Symp., pp. 113-120, 2000.

[12] Chandra, A. and Chakrabarty, K., "Frequency-Directed Run-Length (FDR) Codes with Application to Systems-on-aChip Test Data Compression," Proc. of IEEE VLSI Test Symp., pp. 42-47, 2001.

[13] T. Yamaguchi, M. Tilgner, M. Ishida, and D.S. Ha, “An Efficient Method for Compressing Test Data,” Proc. of Int. Test Conf., pp. 191-199, 1997.

[14] A. Jas and N.A. Touba, "Using an Embedded Processor for Efficient Deterministic Testing of System-on-a-Chip,” Proc. of IEEE Int. Conf. on Computer Design (ICCD), 1999. 